\title{
De klinische relevantie van cribriforme en intraductale prostaatkanker in diagnostische naaldbiopten
}

\author{
Charlotte F. Kweldam ${ }^{1} \cdot$ Intan P. Kümmerlin ${ }^{1}$ - Daan Nieboer ${ }^{2} \cdot$ Esther I. Verhoef $^{1} \cdot$ Ewout W. Steyerberg $^{2}$ • \\ Luca Incrocci $^{3}$ - Chris H. Bangma ${ }^{4}$ Theodorus H. van der Kwast ${ }^{5} \cdot$ Monique J. Roobol $^{4}$ Geert J. van \\ Leenders ${ }^{1}$
}

Published online: 11 January 2017

(C) The Author(s) 2017. This article is available at SpringerLink with Open Access.

Samenvatting Cribriforme en intraductale groei zijn beide geassocieerd met een ongunstige prognose na radicale prostatectomie. In dit onderzoek werden alle patiënten van de eerste screeningsronde van de European Randomized Study of Screening for Prostate Cancer (ERSPC) met prostaatkanker geïncludeerd. Alle uit deze screeningsronde beschikbare coupes werden gereviseerd en gescoord op aanwezigheid van cribriforme en/of intraductale groei. Uitkomstmaten waren biochemisch recidief en ziektespecifieke overleving. In totaal hadden 486 patiënten Gleason-score (GS) 6 (47\%) en 545 GS $\geq 7$ (53\%). De ziektespecifieke en biochemisch-recidiefvrije overleving van CR/IDC--GS3+4-patiënten verschilden niet van die van GS6-patiënten, terwijl die van $\mathrm{CR} / \mathrm{IDC}^{+}$-GS3+4-patiënten wel significant verschillend waren. De conclusie luidt dat de aanwezigheid van cribriforme en intraductale groei bij prostaatkanker een sterke klinische prognostische marker is met veel potentie. Het gebruik van deze variabele bij klinische beslisvorming zou een bijdrage kunnen leveren aan het reduceren van overbehandeling bij prostaatkanker.

drs. Charlotte F. Kweldam

c.kweldam@erasmusmc.nl

1 afdeling Pathologie, Erasmus Medisch Centrum, Rotterdam, Nederland

2 afdeling Maatschappelijke Gezondheidszorg, Erasmus Medisch Centrum, Rotterdam, Nederland

3 afdeling Radiotherapie, Erasmus MC Kanker Instituut, Rotterdam, Nederland

4 afdeling Urologie, Erasmus Medisch Centrum, Rotterdam, Nederland

5 Laboratory Medicine Program, University Health Network, Toronto, Canada
Trefwoorden prostaatkanker $\cdot$ cribriform $\cdot$ intraductaal carcinoom

The clinical relevance of cribriform and intraductal prostate cancer in diagnostic needle biopsies

Abstract Cribriform and intraductal prostate cancer are both associated with an adverse outcome after radical prostatectomy. We included all patients with prostate cancer from the first screening round of the European Randomized Study of Screening for Prostate Cancer (ERSPC). All available slides were revised and scored for presence of cribriform and/or intraductal prostate cancer. Outcome measurements were biochemical recurrence and diseasespecific survival. In total, 486 patients had Gleason score (GS) 6 (47\%) and 545 GS $\geq 7$ (53\%). The disease-specific survival and biochemical-recurrence-free survival of CR/IDC--GS3+4 patients did not differ significantly from those with GS 6, while patients with $\mathrm{CR} / \mathrm{IDC}^{+}-\mathrm{GS} 3+4-$ patients had a significant poorer outcome. Conclusion: The presence of cribriform and intraductal growth in prostate cancer, is a strong clinical prognostic marker with potential. The use of this variable in a clinical setting could contribute to reducing overtreatment of prostate cancer.

Keywords prostate cancer $\cdot$ cribriform $\cdot$ intraductal carcinoma

\section{Introductie}

De zorg omtrent prostaatkanker is uitdagend vanwege de heterogeniteit in histologie, genetica en klinische uitkomst. 
Tabel 1 Patiëntkarakteristieken $(n=1031)$.

\begin{tabular}{|c|c|c|c|c|c|c|}
\hline & $\begin{array}{l}\text { Gleason-score } 6 \\
(n=486)\end{array}$ & $\begin{array}{l}\text { Gleason-score } \\
3+4=7 \\
(n=310)\end{array}$ & $\begin{array}{l}\text { Gleason-score } \\
4+3=7 \\
(n=104)\end{array}$ & $\begin{array}{l}\text { Gleason-score } 8 \\
(n=64)\end{array}$ & $\begin{array}{l}\text { Gleason-score } \\
9-10 \\
(n=67)\end{array}$ & $p$-waarde \\
\hline & \multicolumn{6}{|c|}{ gemiddelde (mediaan, IQR) of $n(\%)$} \\
\hline $\begin{array}{l}\text { PSA-waarde bij diagnose } \\
(\mathrm{ng} / \mathrm{ml})\end{array}$ & $\begin{array}{l}5,8 \\
(4,7 ; 3,5-6,9)\end{array}$ & $\begin{array}{l}8,8 \\
(5,8 ; 4,0-9,0)\end{array}$ & $\begin{array}{l}15 \\
(8,6 ; 4,7-18)\end{array}$ & $\begin{array}{l}19 \\
(11 ; 6,2-17)\end{array}$ & $\begin{array}{l}16 \\
(9,4 ; 5,4-16)\end{array}$ & $<0,001^{*}$ \\
\hline percentage positieve biopten (\%) & $\begin{array}{l}31 \\
(29 ; 17-43)\end{array}$ & $\begin{array}{l}2,9 \\
(3,0 ; 2,0-4,0)\end{array}$ & $\begin{array}{l}50 \\
(43 ; 29-71)\end{array}$ & $\begin{array}{l}55 \\
(50 ; 40-71)\end{array}$ & $\begin{array}{l}62 \\
(57 ; 43-86)\end{array}$ & $<0,001^{*}$ \\
\hline tumorpercentage (\%) & $\begin{array}{l}24 \\
(17 ; 9,5-33)\end{array}$ & $\begin{array}{l}43 \\
(44 ; 27-57)\end{array}$ & $\begin{array}{l}51 \\
(51 ; 33-68)\end{array}$ & $\begin{array}{l}51 \\
(52 ; 33-66)\end{array}$ & $\begin{array}{l}56 \\
(56 ; 41-74)\end{array}$ & $<0,001^{*}$ \\
\hline $\begin{array}{l}C R^{-} / I D C^{+} \text {-status } \\
\text { primaire behandeling }\end{array}$ & $4(0,82)$ & $54(17)$ & $60(58)$ & $33(52)$ & $42(63)$ & $<0,001^{* *}$ \\
\hline radicale prostatectomie & $216(44)$ & $129(42)$ & $33(32)$ & $14(22)$ & $14(21)$ & $<0,001^{* * *}$ \\
\hline radiotherapie & $188(39)$ & $154(59)$ & $66(63)$ & $48(75)$ & $52(78)$ & $<0,001^{* *}$ \\
\hline hormonale therapie & $2(0,41)$ & $3(0,97)$ & $1(0,96)$ & $1(1,6)$ & - & - \\
\hline watchful waiting & $80(17)$ & $23(7,4)$ & $3(2,8)$ & $1(1,6)$ & $1(1,5)$ & $<0,001^{* *}$ \\
\hline $\begin{array}{l}\text { radiotherapie en hormonale } \\
\text { therapie }\end{array}$ & - & - & $1(0,96)$ & - & - & - \\
\hline onbekend & - & $1(0,27)$ & - & - & - & - \\
\hline $\begin{array}{l}\text { prostaatkankergerelateerde } \\
\text { sterfte }\end{array}$ & $8(1,6)$ & $14(4,5)$ & $17(16)$ & $14(22)$ & 19 (28) & - \\
\hline
\end{tabular}

*Kruskal Wallis test.

** Pearson's Chi-square $\left(\chi^{2}\right)$ test.

Vandaag de dag worden klinische beslissingen grotendeels gebaseerd op serum-prostaatspecifiek antigeen (PSA), het klinische tumorstadium en de pathologische Gleason-score (GS) van het biopt. De GS wordt bepaald aan de hand van architectonische tumorgroeipatronen. Patiënten met de laagste GS $(\leq 6)$ hebben een uitstekende uitkomst, terwijl degenen met de hoogste GS (9-10) de ongunstigste prognose hebben [1]. De klinische uitkomst van GS7-patiënten varieert sterk. Door de modificatie van de Gleason-score in 2005 is de GS7-populatie sterk in omvang toegenomen [2]. Ook in de praktijk is GS7 tegenwoordig de meest voorkomende GS, terwijl vóór $2005 \mathrm{GS} 3+3$ dat was. Het verbeteren van de risico-inschatting in de GS7-populatie is relevant, aangezien GS7 de klinische drempelwaarde is voor het starten met actieve behandeling. Recente studies hebben gesuggereerd dat de huidige brede definitie van de Gleason-graad 4-patronen een mogelijke verklaring is voor de variabele behandeluitkomsten bij GS7-prostaatkankerpatiënten [3-6]. Zo is bijvoorbeeld aangetoond dat met name cribriforme groei (een van de graad 4-patronen) in prostatectomieën geassocieerd is met een ongunstige prognose [3-6].

In de afgelopen jaren wordt in toenemende mate de klinische relevantie erkend van het intraductale carcinoom van de prostaat, een hoogrisicolaesie die wordt gedefinieerd als maligne epitheel dat zich uitbreidt in grote acini en ducten, waarbij de basale cellen echter behouden blijven. Het intraductale carcinoom is niet opgenomen in de tumorgradering, maar staat wel bekend om zijn sterke associatie met een hoge GS, een vergevorderd tumorstadium, biochemisch recidief en afstandsmetastasen [7-12]. Het intraductale carcinoom is echter microscopisch zeer lastig te onderscheiden van cribriforme groei, welke invasief is. Voor een dergelijk onderscheid is aanvullende immunohistochemie nodig, waarbij de basale cellen worden aangekleurd. Studies naar de prognostische waarde van intraductaal en cribriform carcinoom zijn grotendeels uitgevoerd op radicale prostatectomiepreparaten [3-6, 11, 13].

In dit artikel bespreken wij eerst twee recente studies van onze groep over de klinische relevantie van cribriforme en intraductale groei bij prostaatkanker. Daarna zullen we onze bevindingen interpreteren en een aantal aanbevelingen doen $[14,15]$.

\section{De prognostische waarde van cribriforme en intraductale groei in prostaatbiopten}

In onze studie vond inclusie plaats van alle 1078 mannen uit de eerste screeningsronde van de European Randomized Study of Screening for Prostate Cancer (ERSPC), die tussen november 1993 en maart 2000 waren gediagnosticeerd met prostaatkanker in het Erasmus Medisch Centrum te Rotterdam $[16,17]$. Exclusiecriteria van de huidige studie waren het ontbreken van coupes of paraffineblokjes ( $n=$ $24)$ en de aanwezigheid van een lymfeklier- of afstandsmetastase bij het stellen van de diagnose $(n=23)$. Uiteindelijk werden 1031 patiënten geschikt bevonden voor verdere 
Figuur 1 Kaplan-Meier-curves voor prostaatkankerspecifieke overleving naar Gleason-score en CR/IDC-status van mannen met Gleason-score 3+4 (a), Gleason-score 4+3 (b), Gleason-score 8 (c) en Gleasonscore 9-10 (d). a

Gleason-score 3+4=7 $(n=310)$

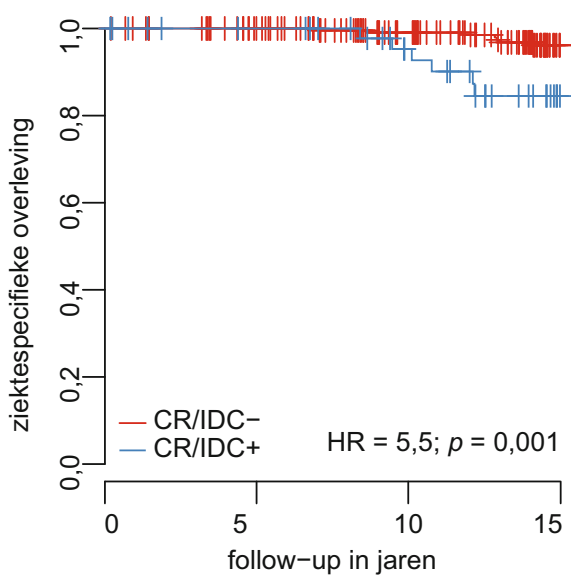

c Gleason-score $8(n=64)$

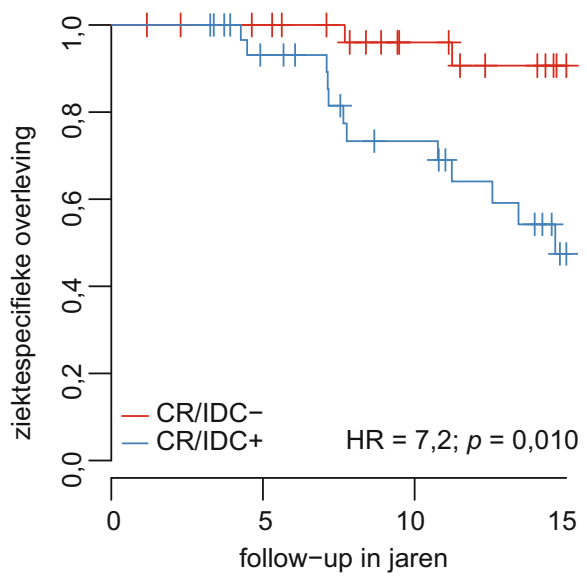

b

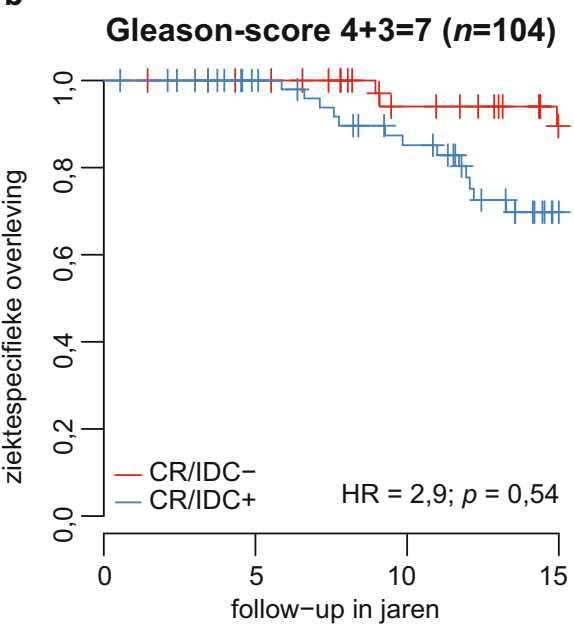

d

Gleason-score 9-10 ( $n=67)$

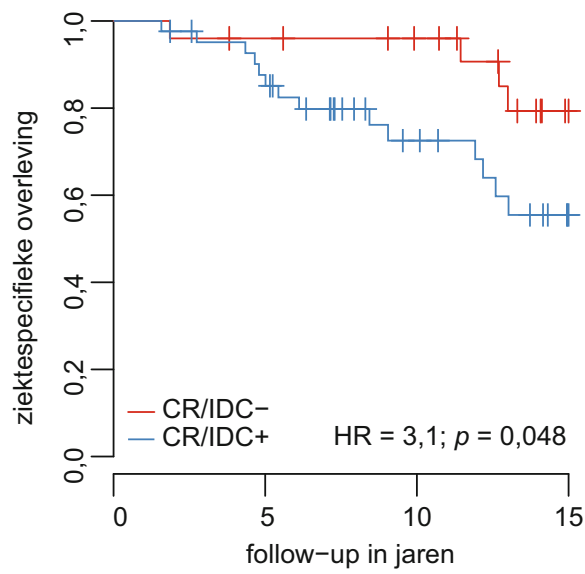

analyse. Drie onderzoekers, die waren geblindeerd voor patiëntgegevens en uitkomst, reviseerden alle histologische coupes. Elk biopt werd gescoord op tumorpercentage, tumorlengte $(\mathrm{mm})$, GS en aanwezigheid van intraductale en of cribriforme groeiwijze [1]. Het label ' $\mathrm{CR} / \mathrm{IDC}^{+}$' werd gegeven aan patiënten die een invasief cribriform carcinoom, een intraductaal carcinoom of beide hadden, en het label ' $\mathrm{CR} / \mathrm{IDC}^{-}$' werd toegekend aan degenen met een prostaatcarcinoom zonder deze kenmerken. Associaties met prostaatkankerspecifieke overleving werden onderzocht met behulp van de Kaplan-Meier-methode, log rank analyse en Cox-regressieanalyse.

De mediane leeftijd van het gehele cohort $(n=1031)$ was 66 jaar (IQR $=62-71)$ en de mediane follow-up was 13 jaar (IQR = 9,4-16; tab. 1). Gedurende de follow-up van het ERSPC-onderzoek waren ten tijde van ons onderzoek in totaal inmiddels 496 patiënten overleden, van wie 72 aan prostaatkanker. Een hogere GS was geassocieerd met leeftijd, PSA-waarde, tumorpercentage en het percentage positieve biopten.
In totaal hadden 193 patiënten een $\mathrm{CR} / \mathrm{IDC}^{+}$-status. De verdeling tussen de GS's is weergegeven in tab. 1 . Slechts vier patiënten hadden een $\mathrm{CR} / \mathrm{IDC}^{+}-\mathrm{GS} 3+3$-status (alle vier met een intraductaal carcinoom). De 15-jaarsprostaatkankerspecifieke overleving van 3+3-patiënten was $99 \%(95 \%$-BI $=97-100)$. De CR/IDC ${ }^{+}$-status was zeer sterk geassocieerd met een slechtere prostaatkankerspecifieke overleving ( $\mathrm{HR}=11 ; 95 \%-\mathrm{BI}=6,6-18 ; p<0,001)$. Bij splitsing van de GS-groepen naar CR/IDC-status was de prostaatkankerspecifieke overleving onder $\mathrm{CR} / \mathrm{IDC}^{+}$patiënten lager dan onder de $\mathrm{CR} / \mathrm{IDC}^{-}$-patiënten (fig. 1). CR/IDC--GS3+4 en GS3+3-patiënten hadden statistisch gelijke overlevingskansen (log rank $p=0,30)$, terwijl onder $\mathrm{CR} / \mathrm{IDC}^{+}-\mathrm{GS} 3+4$-patiënten meer sterfte aan prostaatkanker voorkwam dan onder GS3+3-patiënten ( $\log$ rank $p<$ $0,001)$ en CR/IDC--GS3+4-patiënten (log rank $p=0,001)$. Globaal waren de 15-jaars-prostaatkankerspecifieke overlevingskansen als volgt: $94 \%(95 \%-\mathrm{BI}=91-97)$ onder de patiënten met CR/IDC--GS3+4 of hoger $(n=356)$ en $67 \%(95 \%-\mathrm{BI}=59-76)$ onder de patiënten met $\mathrm{CR} / \mathrm{IDC}^{+}{ }^{+}$ GS3+4 of hoger $(n=189)$. 
Tabel 2 HR's in relatie tot ziektespecifieke overleving in een klinische setting: de toegevoegde waarde van CR/IDC-status, gecorrigeerd voor leeftijd, PSA, percentage positieve biopten, tumorpercentage, Gleason-score en behandeling $(n=1031)$.

\begin{tabular}{|c|c|c|c|c|c|c|}
\hline & model zonc & C-status & & model met & tatus & \\
\hline & HR & $95 \%$-BI & $p$-waarde & HR & $95 \%-B I$ & $p$-waarde \\
\hline leeftijd (jaren) & 0,99 & $0,94-1,0$ & 0,60 & 0,99 & $0,94-1,0$ & 0,63 \\
\hline PSA-waarde ( $\left.\log _{2}\right)$ & $1,2^{\mathrm{a}}$ & $1,0-1,5$ & 0,02 & $1,2^{\mathrm{a}}$ & $1,0-1,5$ & 0,04 \\
\hline percentage positieve biopten ( $\left.\log _{2}\right)$ & $1,8^{\mathrm{a}}$ & $1,2-2,6$ & 0,006 & $1,6^{\mathrm{a}}$ & $1,0-2,4$ & 0,03 \\
\hline tumorpercentage $\left(\log _{2}\right)$ & $1,5^{\mathrm{a}}$ & $1,1-2,1$ & 0,02 & $1,4^{\mathrm{a}}$ & $1,0-2,0$ & 0,05 \\
\hline \multicolumn{7}{|l|}{ Gleason-score } \\
\hline 6 & referentie & & & referentie & & \\
\hline $3+4=7$ & 1,2 & $0,48-3,1$ & .69 & 0,99 & $0,38-2,6$ & 0,99 \\
\hline $4+3=7$ & 3,1 & $1,2-8,0$ & 0,02 & 1,9 & $0,67-5,4$ & 0,23 \\
\hline 8 & 3,7 & $1,4-10$ & 0,01 & 2,3 & $0,78-6,9$ & 0,13 \\
\hline $9-10$ & 5,1 & $2,0-13$ & $<0,001$ & 3,3 & $1,2-9,3$ & 0,02 \\
\hline$C R / I D C^{+}$-status & - & - & - & 2,6 & $1,4-4,8$ & 0,002 \\
\hline radicale prostatectomie & 0,23 & $0,058-0,92$ & 0,04 & 0,26 & $0,064-1,0$ & 0,05 \\
\hline radiotherapie & 1,3 & $0,40-4,5$ & .63 & 1,4 & $0,42-4,7$ & 0,58 \\
\hline
\end{tabular}

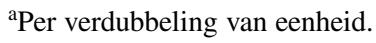

Tabel 3 Patiëntkarakteristieken GS 3+3- en 3+4-cohort $(n=796)$.

\begin{tabular}{|c|c|c|c|c|c|c|c|}
\hline & $\begin{array}{l}\text { GS } 6(n= \\
486)\end{array}$ & $\begin{array}{l}\text { GS } 7(n= \\
310)\end{array}$ & $p$-waarde ${ }^{\mathrm{a}}$ & $\begin{array}{l}\mathrm{GS} \mathrm{7}^{-}(n= \\
256)\end{array}$ & $\begin{array}{l}\text { GS } 7^{+}(n= \\
54)\end{array}$ & $p$-waarde ${ }^{b}$ & $p$-waarde \\
\hline & \multicolumn{3}{|c|}{ gem. (mediaan, IQR) of $n(\%)$} & \multicolumn{4}{|c|}{ gem. (mediaan, IQR) of $n(\%)$} \\
\hline $\begin{array}{l}\text { PSA-waarde bij diagnose } \\
(\mathrm{ng} / \mathrm{ml})\end{array}$ & $\begin{array}{l}5,8 \\
(4,7 ; 3,5-6,9)\end{array}$ & $\begin{array}{l}8,8 \\
(5,8 ; \\
4,0-9,0)\end{array}$ & $<0,001^{*}$ & $\begin{array}{l}8,2 \\
(5,7 ; 4,0-8,4)\end{array}$ & $\begin{array}{l}11 \\
(7,2 ; \\
4,6-12)\end{array}$ & $0,018^{*}$ & $<0,001^{*}$ \\
\hline $\begin{array}{l}\text { percentage positieve biopten } \\
(\%)\end{array}$ & $\begin{array}{l}31 \\
(29 ; 17-43)\end{array}$ & $\begin{array}{l}44 \\
(43 ; 29-57)\end{array}$ & $<0,001^{*}$ & $\begin{array}{l}43 \\
(43 ; 29-57)\end{array}$ & $\begin{array}{l}52 \\
(50 ; 33-67)\end{array}$ & $0,015^{*}$ & $<0,001^{*}$ \\
\hline tumorpercentage (\%) & $\begin{array}{l}24 \\
(17 ; 9,5-33)\end{array}$ & $\begin{array}{l}43 \\
(43 ; 27-57)\end{array}$ & $<0,001^{*}$ & $\begin{array}{l}41 \\
(39 ; 23-56)\end{array}$ & $\begin{array}{l}55 \\
(54 ; 40-66)\end{array}$ & $<0,001^{*}$ & $<0,001^{*}$ \\
\hline \multicolumn{8}{|l|}{ cT-stadium } \\
\hline cT1c & $264(54)$ & $123(40)$ & $<0,001^{* *}$ & $109(42)$ & $14(26)$ & $0,03^{* *}$ & $0,003^{* *}$ \\
\hline cT2 & $181(37)$ & $124(40)$ & $.48^{* *}$ & $104(41)$ & $20(37)$ & $0,74^{* *}$ & $0,41^{* *}$ \\
\hline cT3 & $41(9)$ & $63(20)$ & $<0,001^{* *}$ & 43 (17) & $20(37)$ & $0,002^{* *}$ & $<0,001^{* *}$ \\
\hline \multicolumn{8}{|l|}{ primaire behandeling } \\
\hline radicale prostatectomie & $216(44)$ & $129(42)$ & $0,48^{* *}$ & $112(44)$ & $17(31)$ & $0,13^{* *}$ & $0,92^{* *}$ \\
\hline radiotherapie & $188(39)$ & $154(50)$ & $0,003^{* *}$ & $120(47)$ & $34(63)$ & $0,05^{* *}$ & $0,04^{* *}$ \\
\hline watchful waiting & 80 (16) & $23(7,4)$ & $<0,001^{* *}$ & $20(7,8)$ & $3(5,6)$ & - & $0,002^{* *}$ \\
\hline hormonale behandeling & $2(0,41)$ & $3(0,97)$ & - & $3(1,1)$ & - & - & - \\
\hline onbekend & - & $1(0,32)$ & - & $1(0,39)$ & - & - & - \\
\hline lymfkliermetastase & $2(0,41)$ & $8(2,6)$ & - & $5(2,0)$ & $3(5,6)$ & - & - \\
\hline afstandsmetastase & $9(1,9)$ & $17(5,6)$ & $0,009^{* *}$ & $9(3,5)$ & $8(15)$ & $0,003^{* *}$ & $0,25^{* *}$ \\
\hline
\end{tabular}

GS Gleason-score.

*Mann-Whitney-U-test.

** Pearson's Chi-square $\left(\chi^{2}\right)$ test.

aStatistische vergelijking tussen GS3+3 en $-3+4$.

${ }^{\mathrm{b}}$ Statistische vergelijking tussen $\mathrm{GS}^{-}$en $-7^{+}$.

${ }^{\mathrm{c}}$ Statistische vergelijking tussen GS3+3 en $-7^{-}$. 
Figuur 2 Kaplan-Meier-curves voor biochemisch-recidiefvrije overleving na radicale prostatectomie.

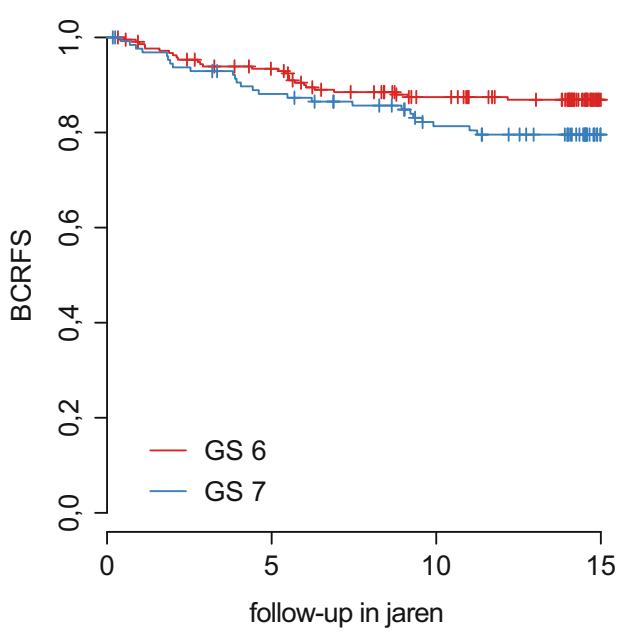

In een multivariabel model analyseerden wij de toegevoegde prognostische waarde van de CR/IDC-status in combinatie met hedendaagse klinisch relevante variabelen, zoals leeftijd, PSA-waarde, behandelingskeuze, GS, tumorpercentage en percentage positieve biopten.

In het model zonder CR/IDC-status waren de volgende variabelen onafhankelijk geassocieerd met een slechtere ziektespecifieke overleving: PSA-waarde, tumorpercentage, percentage positieve biopten en GS4+3 of hoger (tab. 2). Nadat de CR/IDC-status aan het model was toegevoegd, waren GS4+3 en GS8 niet meer onafhankelijk geassocieerd met een slechtere ziektespecifieke overleving.

De conclusie is dus dat de CR/IDC ${ }^{+}$-status in prostaatnaaldbiopten geassocieerd is met een slechtere prostaatkankerspecifieke overleving. Aangezien de prostaatkankerspecifieke overleving van GS3+3- en CR/IDC--GS3+4-patiënten statistisch niet verschillen, zou de CR/IDC-status een waardevolle aanvulling kunnen zijn op de huidige active surveillance criteria.

\section{Uitkomsten van mannen met biopsie GS3+3 en $-3+4$ zonder cribriforme of intraductale groei}

Om de verschillen en overeenkomsten van de hiervoor beschreven GS3+3- $(n=486)$ en $3+4-(n=310)$ populatie verder uit te diepen, hebben we een tweede studie verricht, welke zich concentreert op de tumorkarakteristieken en de tijd tot biochemisch recidief na radicale prostatectomie of radiotherapie. GS3+4-patiënten zonder CR/IDC-groei werden gelabeld als 7-, GS3+4-patiënten met CR/IDC-groei als $7^{+}$. De mediane leeftijd van dit cohort was 66 jaar $(\mathrm{IQR}=62-70)$ en de mediane follow-up 15 jaar $(\mathrm{IQR}=$ 10-17; tab. 3). GS3+4-patiënten hadden significant hogere PSA-waarden $(p<0,001)$ en vaker extracapsulaire groei dan GS3+3-patiënten $(p<0,001)$. Kwantitatieve maten voor tumorgrootte, zoals gemiddeld tumorpercentage $(p<0,001)$ en percentage positieve biopten $(p<0,001)$ waren ook hoger bij GS3+4-patiënten. Alhoewel metastasen spaarzaam waren in dit cohort, hadden mannen met GS3+4 vaker afstandsmetastasen dan mannen met GS3+3 (5,6\% vs. $1,9 \%$; $p<0,009)$. CR/IDC-groei werd gezien bij 54 van de 310 GS3+4-patiënten (17\%). Patiënten met een GS $>7$ hadden hogere PSA-waarden $(p=0,018)$, een groter tumorpercentage $(p<0,001)$ en meer positieve biopten $(p=0,015)$ dan patiënten met een $\mathrm{GS}<7$. Biochemisch recidief werd gezien bij respectievelijk 61 (20\%) GS3+3-, 54 (21\%) GS7-- en $22(41 \%) \mathrm{GST}^{+}$-patiënten.

In de volgende alinea zullen de uitkomsten na radicale prostatectomie en radiotherapie separaat worden besproken.

\section{Het cohort radicale prostatectomie}

In het cohort dat radicale prostatectomie had ondergaan ( $n=345)$ hadden GS3+4-patiënten een ongunstigere biochemisch-recidiefvrije overleving (biochemical-recurrencefree survival; BCRFS) dan degenen met GS3+3 (log rank $p=0,03$; fig. 2). Na het splitsen van de data voor CR/IDCgroei hadden $\mathrm{GS}^{+}{ }^{+}$-patiënten een slechtere BCRFS dan GS3+3- $(\log$ rank $p=0,002)$ en 7--patiënten ( $\log$ rank $p=0,05)$. De BCRFS tussen GS7- en GS3+3-patiënten verschilde niet significant ( $\log$ rank $p=0,13$ ). In een multivariabele analyse waren PSA-waarde en $\mathrm{GS}^{+}$onafhankelijk geassocieerd met een slechtere BCRFS, terwijl GS7- dat niet was (tab. 4).

In het cohort dat radiotherapie had ondergaan $(n=342)$ hadden GS3+4-patiënten een ongunstigere BCRFS dan patiënten met GS3+3 ( $p<0,001$; fig. 3). Na het splitsen voor CR/IDC-groei hadden GS7+-patiënten een slechtere BCRFS dan GS3+3- $(\log$ rank $p<0,001)$ en $7^{-}$-patiënten $(\log$ rank $p=0,01)$. De BCRFS tussen GS3+3- en GS7--patiënten was ook significant verschillend in een ruwe analyse (log rank $p=0,04)$. In de multivariabele analyse waren PSAwaarde, tumorpercentage en percentage positieve biopten 
Hier staat een advertentie.

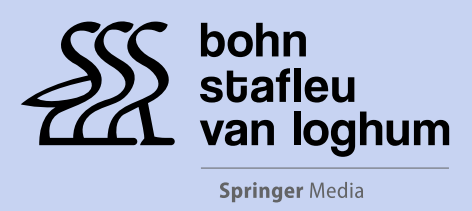

Houten 2017 
Hier staat een advertentie.

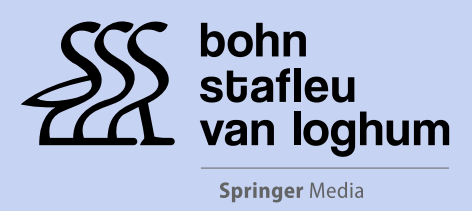

Houten 2017 
Tabel 4 HR's in relatie tot biochemisch recidief na radicale prostatectomie $(n=345)$, gecorrigeerd voor leeftijd, PSA, percentage positieve biopten en tumorpercentage.

\begin{tabular}{llll}
\hline & $\mathrm{HR}$ & $95 \%$-BI & $p$-waarde \\
\hline leeftijd & 1,0 & $0,96-1,1$ & 0,39 \\
PSA & $1,4^{\mathrm{a}}$ & $1,0-2,0$ & 0,03 \\
Gleason-score & & & \\
6 & referentie & & \\
$7^{-}$ & 1,3 & $0,67-2,4$ & 0,47 \\
$7^{+}$ & 3,0 & $1,1-7,8$ & 0,03 \\
tumorpercentage & $0,94^{\mathrm{a}}$ & $0,74-1,2$ & 0,69 \\
$\begin{array}{l}\text { percentage posi- } \\
\text { tieve biopten }\end{array}$ & $1,3^{\mathrm{a}}$ & $0,89-1,9$ & 0,17 \\
\hline
\end{tabular}

aper verdubbeling van eenheid.

Tabel 5 HR's in relatie tot biochemisch recidief na biochemisch recidief na radiotherapie $(n=342)$, gecorrigeerd voor leeftijd, PSA, percentage positieve biopten en tumorpercentage.

\begin{tabular}{llll}
\hline & $\mathrm{HR}$ & $95 \%-\mathrm{BI}$ & $p$-waarde \\
\hline leeftijd & 1,0 & $0,96-1,1$ & 0,71 \\
PSA & $2,2^{\mathrm{a}}$ & $1,8-2,8$ & $<0,001$ \\
Gleason-score & & & \\
6 & referentie & & \\
$7^{-}$ & 0,88 & $0,51-1,5$ & 0,63 \\
$7^{+}$ & 1,2 & $0,58-2,3$ & 0,67 \\
tumorpercentage & $1,4^{\mathrm{a}}$ & $1,1-1,8$ & 0,006 \\
$\begin{array}{l}\text { percentage posi- } \\
\text { tieve biopten }\end{array}$ & $1,4^{\mathrm{a}}$ & $1,1-1,9$ & 0,02 \\
\hline
\end{tabular}

${ }^{\mathrm{a}}$ Verdubbeling van eenheid.

onafhankelijk geassocieerd met een slechtere BCRFS, terwijl GS7- en $\mathrm{GS7}^{+}$dat niet waren (tab. 5).

Concluderend kan worden gesteld dat GS3+4-patiënten, los van CR/IDC-groei, hogere PSA-waarden hebben, een hoger tumorpercentage, een groter percentage positieve biopten en vaker extracapsulaire groei dan patiënten met GS3+3. Patiënten met GS7- hebben geen ongunstigere BCRFS na radicale prostatectomie of radiotherapie dan patiënten met GS $3+3$ en zouden daarom in aanmerking kunnen komen voor active surveillance.

\section{Discussie}

Onze twee studies tonen aan dat CR/IDC-status in diagnostische biopten onafhankelijk geassocieerd is met een slechtere prostaatkankerspecifieke overleving en tijd tot recidief na radicale prostatectomie. De klinisch meest relevante bevinding van deze studies is de uitstekende uitkomst van GS7--patiënten. Hun overleving verschilde niet van de overleving van patiënten met GS6-prostaatkanker. In recente radicale prostatectomiestudies zijn zowel intraductaal als invasieve cribriforme groei geïdentificeerd als onafhankelijke prognostische factoren [3-6, 11, 13]. Tot op heden hebben slechts enkele studies ook gekeken naar de prognostische waarde in diagnostische biopten [7-9]. Deze studies laten zien dat intraductaal carcinoom geassocieerd is met een hoge Gleason-graad en extracapsulaire groei in daaropvolgende radicale prostatectomieën [7, 8]. Daarnaast hebben Kwast et al. aangetoond dat intraductaal carcinoom is geassocieerd met een kortere tijd tot biochemisch recidief en afstandsmetastase na radiotherapie bij intermediair tot hoogrisico-prostaatkankerpatiënten [9].

Alhoewel CR/IDC-status geen directe invloed zal hebben op klinische beslisvorming voor patiënten met een hoge GS, zou de CR/IDC-status wel van waarde kunnen zijn als het gaat om het selecteren van GS3+4-patiënten voor active surveillance dan wel actieve behandeling. Een nadeel van het huidige Gleason-graderingssysteem is de aanzienlijke interobservervariabiliteit, met name bij het maken van onderscheid tussen GS3+3- en GS3+4-prostaatkanker [18-20]. De variabiliteit in gradering is echter voornamelijk gerelateerd aan de aanwezigheid van grillige en gefuseerde groeipatronen; deze betroffen de meerderheid van de GS3+4-prostaatkankers in de huidige studie. Egevad et al. vonden dat cribriforme groei statistisch niet was geassocieerd met interobservervariabiliteit van 337 pathologen [21]. Dit suggereert dat CR/IDC-status een robuustere variabele kan zijn voor risicoschatting dan $\mathrm{GS} 3+3$ of $-3+4$.

Langetermijndata laten zien dat active surveillance voor laagrisicotumoren veilig is, met overlevingspercentages van $98 \%$ na tien jaar en $94 \%$ na 15 jaar [22]. Dit bewijs geldt echter nog niet voor intermediairrisicopatiënten, zoals patiënten met GS 3+4. Ploussard et al. vonden bij 2323 patiënten met een GS3+4-biopt, dat nagenoeg de helft van de patiënten een ongunstige ziekte had na radicale prostatectomie, wat zij definieerden als een pathologische GS $\geq 4+3(21 \%)$, een pT3-4-stadium (37\%) of beide [23]. Bij GS3+4-patiënten die voldeden aan de Prostate Cancer Research International: Active Surveillance (PRIAS) criteria voor active surveillance (cT $\leq \mathrm{T} 2$, PSA $\leq 10 \mathrm{ng} / \mathrm{ml}$, PSAdensiteit $<0,2 \mathrm{ng} / \mathrm{ml} / \mathrm{ml}$ en $\leq 2$ positieve biopten) daalde het aantal patiënten met ongunstige ziekte naar $31 \%$ (19\% GS $\geq 4+3$ en $22 \%$ pT3-4-stadium) [23]. De GS na radicale prostatectomieën bij mannen die voldeden aan de PRIAScriteria steeg naar $\geq 4+3=7$ bij $14-19 \%$ van de mannen en was sprake van extracapsulaire groei bij $10-19 \%$ van de mannen, bij een vijfjaars-BCRFS-ratio van $91 \%$ [24, 25]. De vergelijkbare percentages van opgraderen en opstageren van bovengenoemde GS6- en 7-patiënten komt overeen met onze bevindingen en ondersteunt de rationale om laagrisicoGS7--patiënten te includeren in active surveillance.

Concluderend kan worden gesteld dat de aanwezigheid van cribriforme en intraductale groei bij prostaatkanker een sterke klinische prognostische marker is met veel potentie. 
Figuur 3 Kaplan-Meier-curves voor biochemisch-recidiefvrije overleving na radiotherapie.

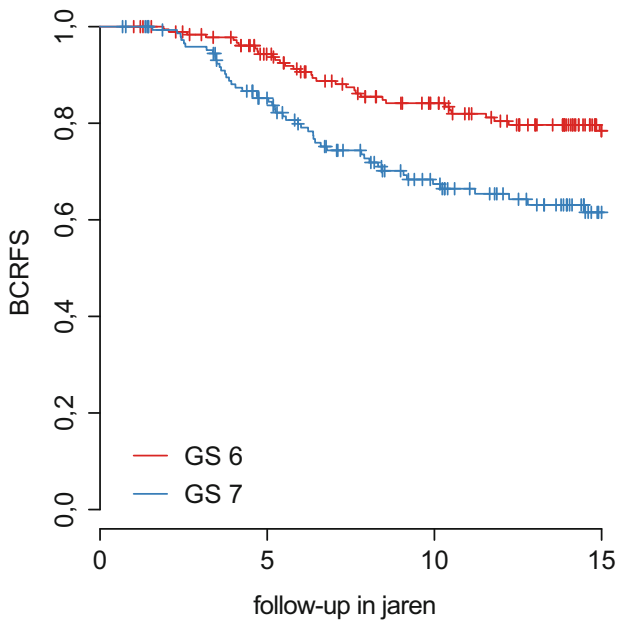

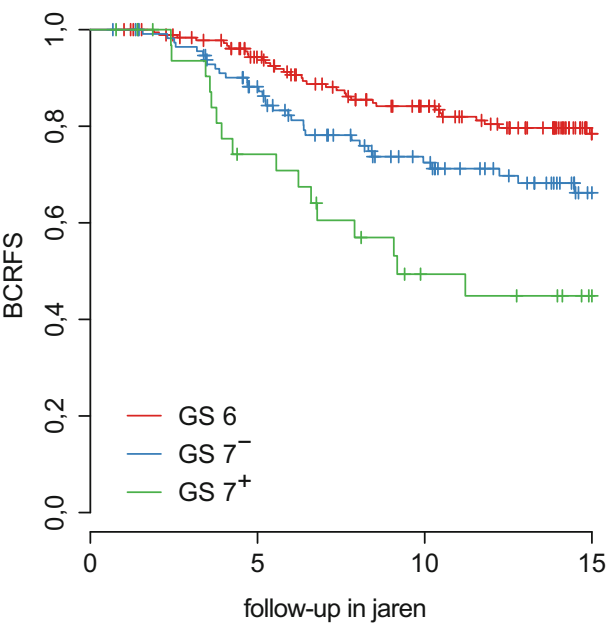

Het gebruik van deze variabele bij klinische beslisvorming zou kunnen bijdragen aan het reduceren van overbehandeling bij prostaatkanker.

\section{Aanbevelingen}

- Vraag uw patholoog de aanwezigheid van cribriforme en intraductale groei specifiek te rapporteren in het verslag.

- Overweeg uw patiënt te includeren voor active surveillance bij een Gleason-score 3+4 zonder cribriforme of intraductale groei, indien de patiënt voldoet aan de overige active surveillance criteria.

Open Access. This article is distributed under the terms of the Creative Commons Attribution 4.0 International License (http:// creativecommons.org/licenses/by/4.0/), which permits unrestricted use, distribution, and reproduction in any medium, provided you give appropriate credit to the original author(s) and the source, provide a link to the Creative Commons license, and indicate if changes were made.

\section{Literatuur}

1. Epstein JI, Egevad L, Amin MB, et al. The 2014 International Society of Urological Pathology (ISUP) Consensus Conference on Gleason Grading of Prostatic Carcinoma: definition of grading patterns and proposal for a new grading system. Am J Surg Pathol. 2016;40(2):244-52.

2. Epstein JI, Allsbrook WC Jr., Amin MB, Egevad LL, Committee IG. The 2005 International Society of Urological Pathology (ISUP) Consensus Conference on Gleason Grading of Prostatic Carcinoma. Am J Surg Pathol. 2005;29(9):1228-42.

3. Iczkowski KA, Torkko KC, Kotnis GR, et al. Digital quantification of five high-grade prostate cancer patterns, including the cribriform pattern, and their association with adverse outcome. Am J Clin Pathol. 2011;136(1):98-107.

4. Kryvenko ON, Gupta NS, Virani N, et al. Gleason score 7 adenocarcinoma of the prostate with lymph node metastases: analysis of 184 radical prostatectomy specimens. Arch Pathol Lab Med. 2013;137(5):610-7.
5. Dong F, Yang P, Wang C, et al. Architectural heterogeneity and cribriform pattern predict adverse clinical outcome for Gleason grade 4 prostatic adenocarcinoma. Am J Surg Pathol. 2013;37(12):185561.

6. Kweldam CF, Wildhagen MF, Steyerberg EW, Bangma CH, Kwast $\mathrm{TH}$ van der, Leenders GJ van. Cribriform growth is highly predictive for postoperative metastasis and disease-specific death in Gleason score 7 prostate cancer. Mod Pathol. 2015;28(3):457-64.

7. Guo CC, Epstein JI. Intraductal carcinoma of the prostate on needle biopsy: Histologic features and clinical significance. Mod Pathol. 2006;19(12):1528-35.

8. Robinson BD, Epstein JI. Intraductal carcinoma of the prostate without invasive carcinoma on needle biopsy: emphasis on radical prostatectomy findings. J Urol. 2010;184(4):1328-33.

9. Kwast T van der, Al Daoud N, Collette L, et al. Biopsy diagnosis of intraductal carcinoma is prognostic in intermediate and high risk prostate cancer patients treated by radiotherapy. Eur J Cancer. 2012;48(9):1318-25.

10. Watts K, Li J, Magi-Galluzzi C, Zhou M. Incidence and clinicopathological characteristics of intraductal carcinoma detected in prostate biopsies: a prospective cohort study. Histopathology. 2013;63(4):574-9.

11. Kimura K, Tsuzuki T, Kato M, et al. Prognostic value of intraductal carcinoma of the prostate in radical prostatectomy specimens. Prostate. 2014;74(6):680-7.

12. Chen ZB, Chen N, Shen PF, et al. The presence and clinical implication of intraductal carcinoma of prostate in metastatic castration resistant prostate cancer. Prostate. 2015;75(12):1247-54.

13. Trudel D, Downes MR, Sykes J, Kron KJ, Trachtenberg J, Kwast TH van der. Prognostic impact of intraductal carcinoma and large cribriform carcinoma architecture after prostatectomy in a contemporary cohort. Eur J Cancer. 2014;50(9):1610-6.

14. Kweldam CF, Kümmerlin IP, Nieboer D, et al. Disease-specific survival of patients with invasive cribriform and intraductal prostate cancer at diagnostic biopsy. Mod Pathol. 2016;29(6):630-6.

15. Kweldam CF, Kummerlin IP, Nieboer D, et al. Prostate cancer outcomes of men with biopsy Gleason score 6 and 7 without cribriform or intraductal carcinoma. Eur J Cancer. 2016;66:26-33.

16. Roobol MJ, Schroder FH. European randomized study of screening for prostate cancer: achievements and presentation. BJU Int. 2003;92(Suppl 2):117-22.

17. Schroder FH, Hugosson J, Roobol MJ, et al. Screening and prostate-cancer mortality in a randomized European study. N Engl J Med. 2009;360(13):1320-8.

18. McKenney JK, Simko J, Bonham M, et al. The potential impact of reproducibility of Gleason grading in men with early stage prostate 
cancer managed by active surveillance: a multi-institutional study. J Urol. 2011;186(2):465-9.

19. Zhou M, Li JB, Cheng L, et al. Diagnosis of 'poorly formed glands' gleason pattern 4 prostatic adenocarcinoma on needle biopsy an interobserver reproducibility study among urologic pathologists with recommendations. Am J Surg Pathol. 2015;39(10):1331-9.

20. Kweldam CF, Nieboer D, Algaba F, et al. Gleason grade 4 prostate adenocarcinoma patterns: an interobserver agreement study among genitourinary pathologists. Histopathology. 2016;69(3):441-9.

21. Egevad L, Algaba F, Berney DM, et al. Interactive digital slides with heat maps: a novel method to improve the reproducibility of Gleason grading. Virchows Arch. 2011;459(2):175-82.

22. Klotz L, Vesprini D, Sethukavalan P, et al. Long-term follow-up of a large active surveillance cohort of patients with prostate cancer. J Clin Oncol. 2015;33(3):272-7.

23. Ploussard G, Isbarn H, Briganti A, et al. Can we expand active surveillance criteria to include biopsy Gleason $3+4$ prostate cancer? A multi-institutional study of 2,323 patients. Urol Oncol. 2015;33(2):71e-79e.

24. Mitsuzuka K, Narita S, Koie T, et al. Pathological and biochemical outcomes after radical prostatectomy in men with low-risk prostate cancer meeting the Prostate Cancer International: Active Surveillance criteria. BJU Int. 2013;111(6):914-20.
25. Bul M, Zhu X, Valdagni R, et al. Active surveillance for lowrisk prostate cancer worldwide: the PRIAS study. Eur Urol. 2013;63(4):597-603.

drs. Charlotte F. Kweldam patholoog in opleiding

dr. Intan P. Kümmerlin patholoog

ir. Daan Nieboer wetenschappelijk onderzoeker

ing. Esther I. Verhoef analist

prof. dr. Ewout W. Steyerberg epidemioloog

prof. dr. Luca Incrocci radiotherapeut

prof. dr. Chris H. Bangma uroloog

prof.dr. Theodorus H. van der Kwast patholoog

dr. Monique J. Roobol epidemioloog

dr. Geert J. van Leenders patholoog 